\title{
A further refinement of Mordell's bound on exponential sums
}

\author{
by \\ Todd Cochrane, Jeremy Coffelt, and \\ Christopher Pinner (Manhattan, KS)
}

1. Introduction. For a prime $p$, integer Laurent polynomial

$$
f(x)=a_{1} x^{k_{1}}+\cdots+a_{r} x^{k_{r}}, \quad p \nmid a_{i}, k_{i} \in \mathbb{Z},
$$

where the $k_{i}$ are distinct and nonzero $\bmod p-1$, and multiplicative character $\chi \bmod p$ we consider the mixed exponential sum

$$
S(\chi, f):=\sum_{x=1}^{p-1} \chi(x) e_{p}(f(x)),
$$

where $e_{p}(\cdot)$ is the additive character $e_{p}(\cdot)=e^{2 \pi i \cdot / p}$ on the finite field $\mathbb{Z}_{p}$. For such sums the classical Weil bound [5] (see [1] or [4] for Laurent $f$ ) yields

$$
|S(\chi, f)| \leq d p^{1 / 2}
$$

where $d$ is the degree of $f$ for a polynomial (degree of the numerator when $f$ has both positive and negative exponents), nontrivial only if $d<\sqrt{p}$. Mordell [3] gave a different type of bound which depended rather on the product of all the exponents $k_{i}$. In [2] we obtained the following improvement in Mordell's bound:

$$
|S(\chi, f)| \leq 4^{1 / r}\left(l_{1} \cdots l_{r}\right)^{1 / r^{2}} p^{1-1 / 2 r},
$$

where

$$
l_{i}= \begin{cases}k_{i} & \text { if } k_{i}>0, \\ r\left|k_{i}\right| & \text { if } k_{i}<0,\end{cases}
$$

nontrivial as long as $\left(l_{1} \cdots l_{r}\right) \leq 4^{-r} p^{r / 2}$. We show here that some of the larger $l_{i}$ can in fact be omitted from the product (at the cost of a worse dependence on $p$ ) once $r \geq 3$ :

2000 Mathematics Subject Classification: 11L07, 11L03.

Key words and phrases: exponential sums. 
THEOREM 1.1. For any $f$ and $\chi$ as above and positive integer $m$ with $r / 2<m \leq r$,

$$
|S(\chi, f)| \leq 4^{1 / m}\left(l_{1} \cdots l_{m}\right)^{1 / m^{2}} p^{1-(m-r / 2) / m^{2}},
$$

where

$$
l_{i}= \begin{cases}k_{i} & \text { if } k_{i}>0 \\ m\left|k_{i}\right| & \text { if } k_{i}<0\end{cases}
$$

The theorem thus implies a nontrivial bound on $|S(\chi, f)|$ as long as $\left(l_{1} \cdots l_{m}\right)<4^{-m} p^{m-r / 2}$ for some $r / 2<m \leq r$. Inequality (1.3) is just the case $m=r$. One can in fact save an extra factor of $\left(\left(k_{1}, \ldots, k_{r}, p-1\right) /\right.$ $\left.\left(k_{1}, \ldots, k_{m}\right)\right)^{1 / m^{2}}$ on the stated bound, as we explain in Section 2 below. Theorem 1.1 is particularly useful when more than half of the exponents are small; in particular (for fixed $r$ ) if at least $R=\lfloor r / 2\rfloor+1$ of the $k_{i}$ are bounded, $l_{i} \leq B$ say, then one obtains a uniform bound

$$
|S(\chi, f)| \leq(4 B)^{1 / R} p^{1-\delta}
$$

with $\delta=1 / R^{2}$ or $1 / 2 R^{2}$ as $r$ is even or odd, irrespective of the size of the remaining $l_{i}$. Notice one cannot expect a bound of order $p^{1-\delta}$ with some $\delta>0$ if only $\lfloor r / 2\rfloor$ of the $k_{i}$ are bounded as can be seen for the sums $|S(\chi, f)|=p / 2+O(r \sqrt{p})$ when

$$
f=\varepsilon a_{0} x^{(p-1) / 2}+\sum_{i=1}^{\lfloor r / 2\rfloor} a_{i}\left(x^{i}-x^{i+(p-1) / 2}\right), \quad \chi(x)=\chi_{0}(x) \text { or }\left(\frac{x}{p}\right),
$$

with $\varepsilon=0$ or 1 as $r$ is even or odd.

For monomials and binomials we gain nothing new, but for trinomials

$$
f=a x^{k_{1}}+b x^{k_{2}}+c x^{k_{3}}
$$

we obtain the $m=2$ Theorem 1.1 bound

$$
|S(\chi, f)| \leq\left(k_{1} k_{2}\right)^{1 / 4} p^{7 / 8}
$$

avoiding entirely the need to involve the largest exponent, in contrast to the Weil bound and our previous Mordell type bound $(m=3)$ :

$$
|S(\chi, f)| \leq \max \left\{k_{1}, k_{2}, k_{3}\right\} p^{1 / 2}, \quad|S(\chi, f)| \leq \sqrt[9]{80 / 9}\left(k_{1} k_{2} k_{3}\right)^{1 / 9} p^{5 / 6} .
$$

The proof of the theorem is very similar to that of (1.3) and involves bounding the number of solutions $\left(x_{1}, \ldots, x_{m}, y_{1}, \ldots, y_{m}\right)$ in $\mathbb{Z}_{p}^{* 2 m}$ to the system of simultaneous equations

$$
x_{1}^{k_{i}}+\cdots+x_{m}^{k_{i}} \equiv y_{1}^{k_{i}}+\cdots+y_{m}^{k_{i}} \bmod p
$$

for $i=1, \ldots, r$. We denote the number of such solutions by $M_{m}$. For $m \leq r$ we can merely use the first $m$ equations (discarding the remaining $r-m$ ) and appeal to the bound of Mordell [3] or Lemma 3.1 in [2] to obtain:

$$
M_{m} \leq 4^{m}\left(l_{1} \cdots l_{m}\right)(p-1)^{m} .
$$


The theorem is then immediate from (1.8) by taking $v=w=m$ in the following lemma relating $S(\chi, f)$ to $M_{m}$ :

Lemma 1.1. For any $f$ and $\chi$ as above, and positive integers $v, w$,

$$
|S(\chi, f)| \leq(p-1)^{1-1 / v-1 / w} p^{r / 2 v w}\left(M_{v} M_{w}\right)^{1 / 2 v w} .
$$

2. Slight improvements in the bound for $M_{m}$. Although it seems wasteful to simply discard the remaining $r-m$ equations in (1.7) there are certainly cases where these equations are redundant. For instance, if the first $m$ exponents take the form $k_{i}=i l, i=1, \ldots, m$, with $l \mid k_{i}$ for the remaining $k_{i}$ then the $x_{i}^{l}$ are merely a permutation of the $y_{i}^{l}$ whatever those remaining exponents. Moreover when $m=2$ our [2] bound for the first two equations

$$
M_{2} \leq \begin{cases}k_{1} k_{2}(p-1)^{2} & \text { if } k_{1} k_{2}>0 \\ 3\left|k_{1} k_{2}\right|(p-1)^{2} & \text { if } k_{1} k_{2}<0\end{cases}
$$

can be asymptotically sharp; for example for exponents $k_{1}=l, k_{2}=2 l$, with $l \mid k_{i}, i=3, \ldots, r$, and $l \mid(p-1)$ or $k_{1}=l, k_{2}=-l$ or $3 l$ and $l \mid k_{i}, i=3, \ldots, r$, with the $k_{i} / l$ odd and $2 l \mid(p-1)$, it is not hard to see that

$$
\begin{aligned}
& M_{2}=2 l^{2}(p-1)^{2}-l^{3}(p-1), \\
& M_{2}=3 l^{2}(p-1)^{2}-3 l^{3}(p-1),
\end{aligned}
$$

respectively. In certain cases though we can utilize the remaining equations for a slight saving:

Lemma 2.1. If $r \geq 2$ and

$$
L_{i j}= \begin{cases}k_{i} k_{j} & \text { if } k_{i} k_{j}>0 \\ 3\left|k_{i} k_{j}\right| & \text { if } k_{i} k_{j}<0\end{cases}
$$

then for $m=2$ we have

$$
M_{2} \leq\left(k_{1}, \ldots, k_{r}, p-1\right) \min _{1 \leq i<j \leq r} \frac{L_{i j}}{\left(k_{i}, k_{j}\right)}(p-1)^{2} .
$$

Thus for example in the trinomial case (1.6) can be slightly refined to

$$
|S(\chi, f)| \leq\left(\frac{\left(k_{1}, k_{2}, k_{3}, p-1\right)}{\left(k_{1}, k_{2}\right)}\right)^{1 / 4}\left(k_{1} k_{2}\right)^{1 / 4} p^{7 / 8},
$$

of use if $k_{1}$ and $k_{2}$ share a common factor not shared with $k_{3}$. More generally a slight modification of the proof of Lemma 3.1 in [2] allows a similar saving of a factor $\left(k_{1}, \ldots, k_{r}, p-1\right) /\left(k_{1}, \ldots, k_{m}\right)$ on the previous bound (1.8):

LEMMA 2.2. If $r \geq 3$, then for any $3 \leq m \leq r$ and choice of $m$ exponents $k_{1}, \ldots, k_{m}$,

$$
M_{m} \leq \frac{4 e}{m^{2}}\left(\begin{array}{c}
2 m \\
m
\end{array}\right) \frac{\left(k_{1}, \ldots, k_{r}, p-1\right)}{\left(k_{1}, \ldots, k_{m}\right)}\left(l_{1} \cdots l_{m}\right)(p-1)^{m} .
$$


3. Proof of Lemma 1.1. For $\vec{u}=\left(u_{1}, \ldots, u_{r}\right) \in \mathbb{Z}_{p}^{r}$ and positive integer $m$, we define

$$
N_{m}(\vec{u})=\#\left\{\left(x_{1}, \ldots, x_{m}\right) \in \mathbb{Z}_{p}^{* m}: \sum_{i=1}^{m} x_{i}^{k_{j}}=u_{j}, j=1, \ldots, r\right\},
$$

and observe that

$$
\sum_{\vec{u} \in \mathbb{Z}_{p}^{r}} N_{m}(\vec{u})=(p-1)^{m}, \quad \sum_{\vec{u} \in \mathbb{Z}_{p}^{r}} N_{m}^{2}(\vec{u})=M_{m} .
$$

For any multiplicative character $\chi$ and positive integer $m$, the simple observation that $\sum_{u \in \mathbb{Z}_{p}} e_{p}(a u)=p$ if $a \equiv 0 \bmod p$ and zero otherwise gives

$$
\begin{aligned}
\sum_{\vec{u} \in \mathbb{Z}_{p}^{r}}\left|\sum_{x=1}^{p-1} \chi(x) e_{p}\left(a_{1} u_{1} x^{k_{1}}+\cdots+a_{r} u_{r} x^{k_{r}}\right)\right|^{2 m} & \\
= & \sum_{\substack{x_{1}, \ldots, x_{m}, y_{1}, \ldots, y_{m} \in \mathbb{Z}_{p}^{*}}} \chi\left(x_{1} \cdots x_{m} y_{1}^{-1} \cdots y_{m}^{-1}\right) \\
& \times \sum_{\vec{u} \in \mathbb{Z}_{p}^{r}} e_{p}\left(\sum_{j=1}^{r} a_{j} u_{j}\left(x_{1}^{k_{j}}+\cdots+x_{m}^{k_{j}}-y_{1}^{k_{j}}-\cdots-y_{m}^{k_{j}}\right)\right) \\
= & p^{r} \sum^{*} \chi\left(x_{1} \cdots x_{m} y_{1}^{-1} \cdots y_{m}^{-1}\right) \leq p^{r} M_{m},
\end{aligned}
$$

where $\sum^{*}$ denotes a sum over the $x_{1}, \ldots, x_{m}, y_{1}, \ldots, y_{m}$ in $\mathbb{Z}_{p}^{*}$ satisfying $\sum_{j=1}^{m} x_{j}^{k_{i}} \equiv \sum_{j=1}^{m} y_{j}^{k_{i}} \bmod p$ for $1 \leq i \leq r$.

Writing $S=S(\chi, f)$, we have

$$
\begin{aligned}
(p-1) S^{w} & =\sum_{m=1}^{p-1}\left(\sum_{x=1}^{p-1} \chi(m x) e_{p}\left(a_{1}(m x)^{k_{1}}+\cdots+a_{r}(m x)^{k_{r}}\right)\right)^{w} \\
& =\sum_{m=1}^{p-1} \chi^{w}(m) \sum_{x_{1}, \ldots, x_{w} \in \mathbb{Z}_{p}^{*}} \chi\left(x_{1} \cdots x_{w}\right) e_{p}\left(\sum_{j=1}^{r} a_{j} m^{k_{j}}\left(x_{1}^{k_{j}}+\cdots+x_{w}^{k_{j}}\right)\right) \\
& =\sum_{x_{1}, \ldots, x_{w} \in \mathbb{Z}_{p}^{*}} \chi\left(x_{1} \cdots x_{w}\right) \sum_{m=1}^{p-1} \chi^{w}(m) e_{p}\left(\sum_{j=1}^{r} a_{j} m^{k_{j}}\left(x_{1}^{k_{j}}+\cdots+x_{w}^{k_{j}}\right)\right),
\end{aligned}
$$

and so

$$
(p-1)|S|^{w} \leq \sum_{\vec{u} \in \mathbb{Z}_{p}^{r}} N_{w}(\vec{u})\left|\sum_{m=1}^{p-1} \chi^{w}(m) e_{p}\left(\sum_{j=1}^{r} a_{j} u_{j} m^{k_{j}}\right)\right| .
$$

Applying Hölder's inequality twice, the second time splitting

$$
N_{w}(\vec{u})^{2 v /(2 v-1)}=N_{w}(\vec{u})^{(2 v-2) /(2 v-1)} N_{w}(\vec{u})^{2 /(2 v-1)},
$$


and using (3.1) and (3.2) gives

$$
\begin{aligned}
(p-1)|S|^{w} \leq & \left(\sum_{\vec{u}} N_{w}(\vec{u})^{2 v /(2 v-1)}\right)^{(2 v-1) / 2 v} \\
& \times\left(\sum_{\vec{u}}\left|\sum_{m=1}^{p-1} \chi^{w}(m) e_{p}\left(a_{1} u_{1} m^{k_{1}}+\cdots+a_{r} u_{r} m^{k_{r}}\right)\right|^{2 v}\right)^{1 / 2 v} \\
\leq & \left(\left(\sum_{\vec{u}} N_{w}(\vec{u})\right)^{(2 v-2) /(2 v-1)}\right. \\
& \left.\times\left(\sum_{\vec{u}} N_{w}^{2}(\vec{u})\right)^{1 /(2 v-1)}\right)^{(2 v-1) / 2 v}\left(M_{v} p^{r}\right)^{1 / 2 v} \\
= & \left((p-1)^{w}\right)^{(v-1) / v}\left(M_{w}\right)^{1 / 2 v}\left(M_{v} p^{r}\right)^{1 / 2 v} \\
= & (p-1)^{w(1-1 / v)} p^{r / 2 v}\left(M_{v} M_{w}\right)^{1 / 2 v} .
\end{aligned}
$$

Hence

$$
|S|<(p-1)^{1-1 / v-1 / w} p^{r / 2 v w}\left(M_{v} M_{w}\right)^{1 / 2 v w}
$$

4. Proof of Lemma 2.1. Write $M_{2}=\sum_{\vec{u} \in \mathbb{Z}_{p}^{r}} C(\vec{u})^{2}$ where

$$
\begin{aligned}
C\left(u_{1}, \ldots, u_{r}\right) & =\#\left\{(x, y) \in \mathbb{Z}_{p}^{* 2}: x^{k_{i}}-y^{k_{i}}=u_{i} \text { for } i=1, \ldots, r\right\} \\
& =d \#\left\{x \in \mathbb{Z}_{p}^{*}: \exists y \in \mathbb{Z}_{p}^{*} \text { with } x^{k_{i}}-y^{k_{i}}=u_{i} \text { for } i=1, \ldots, r\right\},
\end{aligned}
$$

and $d=\left(k_{1}, \ldots, k_{r}, p-1\right)$ (since for each $x$ with a solution $y_{0}$ there will be $d$ solutions $y$ satisfying $\left.y^{\left(k_{1}, \ldots, k_{r}\right)}=y_{0}^{\left(k_{1}, \ldots, k_{r}\right)}\right)$. Note the trivial bound $C(\vec{u}) \leq d(p-1)$.

If $0<k_{1}<k_{2}$ and $\left(u_{1}, u_{2}\right) \neq(0,0)$ then any $x$ in the latter set must be a root of the nonzero polynomial

$$
f=\left(x^{k_{1}}-u_{1}\right)^{k_{2} /\left(k_{1}, k_{2}\right)}-\left(x^{k_{2}}-u_{2}\right)^{k_{1} /\left(k_{1}, k_{2}\right)},
$$

which has degree at most $k_{1}\left(k_{2} /\left(k_{1}, k_{2}\right)-1\right)$, and so

$$
C(\vec{u}) \leq \frac{d k_{1} k_{2}}{\left(k_{1}, k_{2}\right)}-d k_{1} .
$$

On the other hand, if $k_{1}<0<k_{2}$ and $\left(u_{1}, u_{2}\right) \neq(0,0)$ then $x$ will be a root of the nonzero polynomial

$$
f=\left(x^{k_{2}}-u_{2}\right)^{\left|k_{1}\right| /\left(k_{1}, k_{2}\right)}\left(1-u_{1} x^{\left|k_{1}\right|}\right)^{k_{2} /\left(k_{1}, k_{2}\right)}-x^{\left|k_{1}\right| k_{2} /\left(k_{1}, k_{2}\right)},
$$

of degree at most $2\left|k_{1}\right| k_{2} /\left(k_{1}, k_{2}\right)$, and so

$$
C(\vec{u}) \leq 2 \frac{d}{\left(k_{1}, k_{2}\right)}\left|k_{1}\right| k_{2} .
$$


Now for $\left(u_{1}, u_{2}\right)=(0,0)$, we will evaluate the sum $\sum_{\left(u_{1}, u_{2}\right)=(0,0)} C(\vec{u})$. Since $x^{k_{1}}=y^{k_{1}}$ and $x^{k_{2}}=y^{k_{2}}$ imply $x^{\left(k_{1}, k_{2}\right)}=y^{\left(k_{1}, k_{2}\right)}$, we have

$$
\begin{aligned}
& \sum_{\left(u_{1}, u_{2}\right)=(0,0)} C(\vec{u}) \\
= & \sum_{\left(u_{1}, u_{2}\right)=(0,0)} \#\left\{(x, y) \in \mathbb{Z}_{p}^{* 2}: x^{\left(k_{1}, k_{2}\right)}=y^{\left(k_{1}, k_{2}\right)}, x^{k_{l}}-y^{k_{l}}=u_{l} \text { for } l \neq 1,2\right\} \\
= & \#\left\{(x, y) \in \mathbb{Z}_{p}^{* 2}: x^{\left(k_{1}, k_{2}\right)}=y^{\left(k_{1}, k_{2}\right)}\right\}=\left(k_{1}, k_{2}, p-1\right)(p-1) .
\end{aligned}
$$

Finally, since $\sum_{\vec{u} \in \mathbb{Z}_{p}^{r}} C(\vec{u})=(p-1)^{2}$, for $0<k_{1}<k_{2}$ we have

$$
\begin{aligned}
M_{2}= & \sum_{\left(u_{1}, u_{2}\right) \neq(0,0)} C(\vec{u})^{2}+\sum_{\left(u_{1}, u_{2}\right)=(0,0)} C(\vec{u})^{2} \\
\leq & \left(\frac{d k_{1} k_{2}}{\left(k_{1}, k_{2}\right)}-d k_{1}\right) \sum_{\left(u_{1}, u_{2}\right) \neq(0,0)} C(\vec{u})+d(p-1) \sum_{\left(u_{1}, u_{2}\right)=(0,0)} C(\vec{u}) \\
= & \left(\frac{d k_{1} k_{2}}{\left(k_{1}, k_{2}\right)}-d\left(k_{1}-\left(k_{1}, k_{2}, p-1\right)\right)\right)(p-1)^{2} \\
& -\left(k_{1}, k_{2}, p-1\right)\left(\frac{d k_{1} k_{2}}{\left(k_{1}, k_{2}\right)}-d k_{1}\right)(p-1) \\
< & d \frac{k_{1} k_{2}}{\left(k_{1}, k_{2}\right)}(p-1)^{2},
\end{aligned}
$$

and for $k_{1}<0<k_{2}$,

$$
\begin{aligned}
M_{2}= & \sum_{\left(u_{1}, u_{2}\right) \neq(0,0)} C(\vec{u})^{2}+\sum_{\left(u_{1}, u_{2}\right)=(0,0)} C(\vec{u})^{2} \\
\leq & 2 \frac{d}{\left(k_{1}, k_{2}\right)}\left|k_{1}\right| k_{2} \sum_{\left(u_{1}, u_{2}\right) \neq(0,0)} C(\vec{u})+d(p-1) \sum_{\left(u_{1}, u_{2}\right)=(0,0)} C(\vec{u}) \\
= & \left(2 \frac{d}{\left(k_{1}, k_{2}\right)}\left|k_{1}\right| k_{2}+d\left(k_{1}, k_{2}, p-1\right)\right)(p-1)^{2} \\
& -2 \frac{d}{\left(k_{1}, k_{2}\right)}\left(k_{1}, k_{2}, p-1\right)\left|k_{1}\right| k_{2}(p-1) \\
< & 3 \frac{d}{\left(k_{1}, k_{2}\right)}\left|k_{1}\right| k_{2}(p-1)^{2} .
\end{aligned}
$$

Since the proof holds when the $k_{i}$ 's are interchanged, we have the desired result.

5. Proof of Lemma 2.2. The proof is almost identical to that of Lemma 3.1 in [2]. Simply ignore the $r-m$ remaining equations for all of 
the proof except for the instance where Wooley's result [6] was applied to bound the number of solutions to

$$
u_{1}^{k_{j}}+u_{2}^{k_{j}}+\cdots+u_{t}^{k_{j}}=\alpha_{j} \quad \text { for } j=1, \ldots, t,
$$

for some $1 \leq t \leq m$ with $D_{t}(\vec{u}) \neq 0$. Instead of bounding the number of solutions to the above system, bound the number of solutions to

$$
X_{1}^{k_{j} / d}+X_{2}^{k_{j} / d}+\cdots+X_{t}^{k_{j} / d}=\alpha_{j} \quad \text { for } j=1, \ldots, t
$$

where $d=\left(k_{1}, k_{2}, \ldots, k_{m}\right)$ and $X_{i}=u_{i}^{d}$. By the previously mentioned result of Wooley, we know that the number of solutions to the second system is no more than $\left(k_{1} / d\right)\left(k_{2} / d\right) \cdots\left(k_{t} / d\right)$. However, for a given value of $X_{i}$ there are at most $(d, p-1)$ values for $u_{i}$ such that $u_{i}^{d}=X_{i}$. After fixing values for all but one of the $u_{i}$, say $u_{1}$, the values $u_{1}^{k_{1}}, \ldots, u_{1}^{k_{r}}$ are all determined, so that the number of choices for $u_{1}$ is at most $\left(k_{1}, \ldots, k_{r}, p-1\right)$. This gives no more than

$$
\left(k_{1}, \ldots, k_{r}, p-1\right)(d, p-1)^{t-1}\left(k_{1} / d\right) \cdots\left(k_{t} / d\right) \leq \frac{\left(k_{1}, \ldots, k_{r}, p-1\right)}{d} k_{1} \cdots k_{t}
$$

solutions, improving on the previous bound of $k_{1} \cdots k_{t}$ (given by the direct application of Wooley's result on only the first $t$ equations) by the desired factor.

\section{References}

[1] F. N. Castro and C. J. Moreno, Mixed exponential sums over finite fields, Proc. Amer. Math. Soc. 128 (2000), 2529-2537.

[2] T. Cochrane and C. Pinner, An improved Mordell type bound for exponential sums, Proc. Amer. Math. Soc., to appear.

[3] L. J. Mordell, On a sum analogous to a Gauss's sum, Quart. J. Math. Oxford Ser. 3 (1932), 161-167.

[4] G. I. Perel'muter, Estimate of a sum along an algebraic curve, Mat. Zametki 5 (1969), 373-380 (in Russian).

[5] A. Weil, On some exponential sums, Proc. Nat. Acad. Sci. U.S.A. 34 (1948), 204-207.

[6] T. Wooley, A note on simultaneous congruences, J. Number Theory 58 (1996), 288297.

Department of Mathematics

Kansas State University

Manhattan, KS 66506, U.S.A.

E-mail: cochrane@math.ksu.edu jcoffelt@math.ksu.edu pinner@math.ksu.edu 